Trauma Berufskrankh 2014 · 16[Suppl 2]: 173-179 DOI 10.1007/s10039-013-2018-6

Online publiziert: 16. Oktober 2013

(c) Springer-Verlag Berlin Heidelberg 2013
H.J. Bail ${ }^{1} \cdot$ K. van Scherpenzeel ${ }^{2}$

${ }^{1}$ Klinik für Unfall- und Orthopädische Chirurgie, Klinikum Nürnberg

${ }^{2}$ Centrum für Muskuloskeletale Chirurgie, Charité Universitätsmedizin Berlin,

Campus Virchow-Klinikum, Berlin

\title{
Rekonstruktion von posttraumatischen Beindeformitäten
}

Die Gründe für Fehlstellungen der unteren Extremitäten sind vielfältig: Stoffwechselerkrankungen, benigne und maligne Tumoren, genetische Defekte und verschiedene Arten von Mineralisierungsstörungen können Ursache für Achsabweichungen, Verkürzungen oder übermäBiges Wachstum der unteren Extremitäten sein. Im vorliegenden Beitrag werden die posttraumatischen Fehlstellungen als die häufigsten erworbenen Fehlstellungen behandelt. Es wird v. a. auf die Indikation zur Fehlstellungskorrektur und hinsichtlich der Methoden insbesondere auf die Korrektur mit dem Hexapoden und mit dem intramedullären Verlängerungsnagel eingegangen.

\section{Diagnostik}

Es ist wichtig, die normalen Verhältnisse mit ihren Standardabweichungen zu kennen. Bei den posttraumatischen Veränderungen hilft der Seitenvergleich, wenn nur eine Extremität betroffen ist. Maßgebend sind die sog. Gelenkwinkel, wie sie von Paley [6] und Strecker [8] formuliert wurden. Wichtig ist, bei der Achsanalyse immer sowohl die anatomischen als auch die mechanischen Achsen und Winkel zu berücksichtigen. Hinsichtlich der anatomischen Achse sind die folgenden Fehlstellungen zu definieren: Varus/Valgus (ad axim), Verkürzung/Verlängerung (ad longitudinem cum contractionem/distractionem), Translation (ad latus) und Torsion (ad peripheriam).
Klinik

Um die jeweiligen Achsen und die klinischen Auswirkungen der jeweiligen Fehlstellung zu überprüfen, ist zunächst eine gründliche klinische Untersuchung erforderlich. Hierbei werden die Symmetrie und die Achsen im Stand überprüft und die Länge der Beine im Stehen mit Hilfe von entsprechenden Unterlegplatten bestimmt. Dabei gilt es maßgeblich, die Beckenkippung in der seitlichen und der Frontalebene zu beachten, Gesäßfalten werden auf Symmetrie überprüft, und eine begleitende oder auch ursächliche Skoliose der Wirbelsäule muss Berücksichtigung finden.

Die Längen von Ober- und Unterschenkel werden zur Differenzierung, ob eine Beinlängendifferenz femoral oder tibial begründet ist, im Liegen und Sitzen untersucht. Die Hüftrotation wird im Sitzen und hauptsächlich hinweisgebend in Bauchlage geprüft. Die Rotation der Tibia wird in $90^{\circ}$-Stellung des Kniegelenks und des OSG (oberes Sprunggelenk) kontrolliert, zusätzlich in Rückenlage und $90^{\circ}$ Beugung im Hüft-, Knie- und Sprunggelenk. Hierbei gilt es zu beachten, dass die Gelenke frei beweglich sein müssen, es dürfen keine Bandinstabilitäten vorliegen, um evtl. pathologische Rotationsfehlstellungen beurteilen zu können.

\section{Bildgebung}

Sie ist von elementarer Bedeutung: Neben den Aufnahmen der betroffenen Skelettabschnitte in 2 Ebenen mit angrenzenden Gelenken müssen auch standardisierte Becken-Bein-Ganzaufnahmen im Stehen erstellt werden. Die Becken-BeinGanzaufnahme im Liegen kann in den meisten Fällen unterbleiben, da für viele Fehlstellungen ohnehin eine CT-Untersuchung (CT: Computertomographie) notwendig ist.

In der frontalen 2D-Rekonstruktion oder im Übersichtsscan können die Achsverhältnisse im Liegen ausgemessen wer-

\begin{tabular}{|ll}
\hline Tab. 1 Für die Indikationsstellen wesentliche Maßzahlen \\
\hline Frontalebene & $\begin{array}{l}\text { Varus }>5-10^{\circ} \\
\text { Valgus }>10^{\circ}\end{array}$ \\
\hline Sagittalebene & $\begin{array}{l}\text { Ossäre Streckdefizite }>5-10^{\circ} \\
\text { Diaphysäre Deformität }>15^{\circ} \\
\text { Metaphysäre und epiphysäre Fehlstellungen 5-10 (in der Sagittalebene } \\
\text { ist immer das funktionelle Defizit entscheidend) }\end{array}$ \\
\hline Transversalebene & Gelenknahe Deformität \\
\hline Längendifferenz & $>2$ cm beim jungen Patienten (Oberschenkel:Unterschenkel=5:4) \\
\hline Torsion & $>15^{\circ}$ \\
& Fehlen des rotatorischen 0-Durchgangs \\
\hline
\end{tabular}




\section{Innovation/Außenseitermethoden}
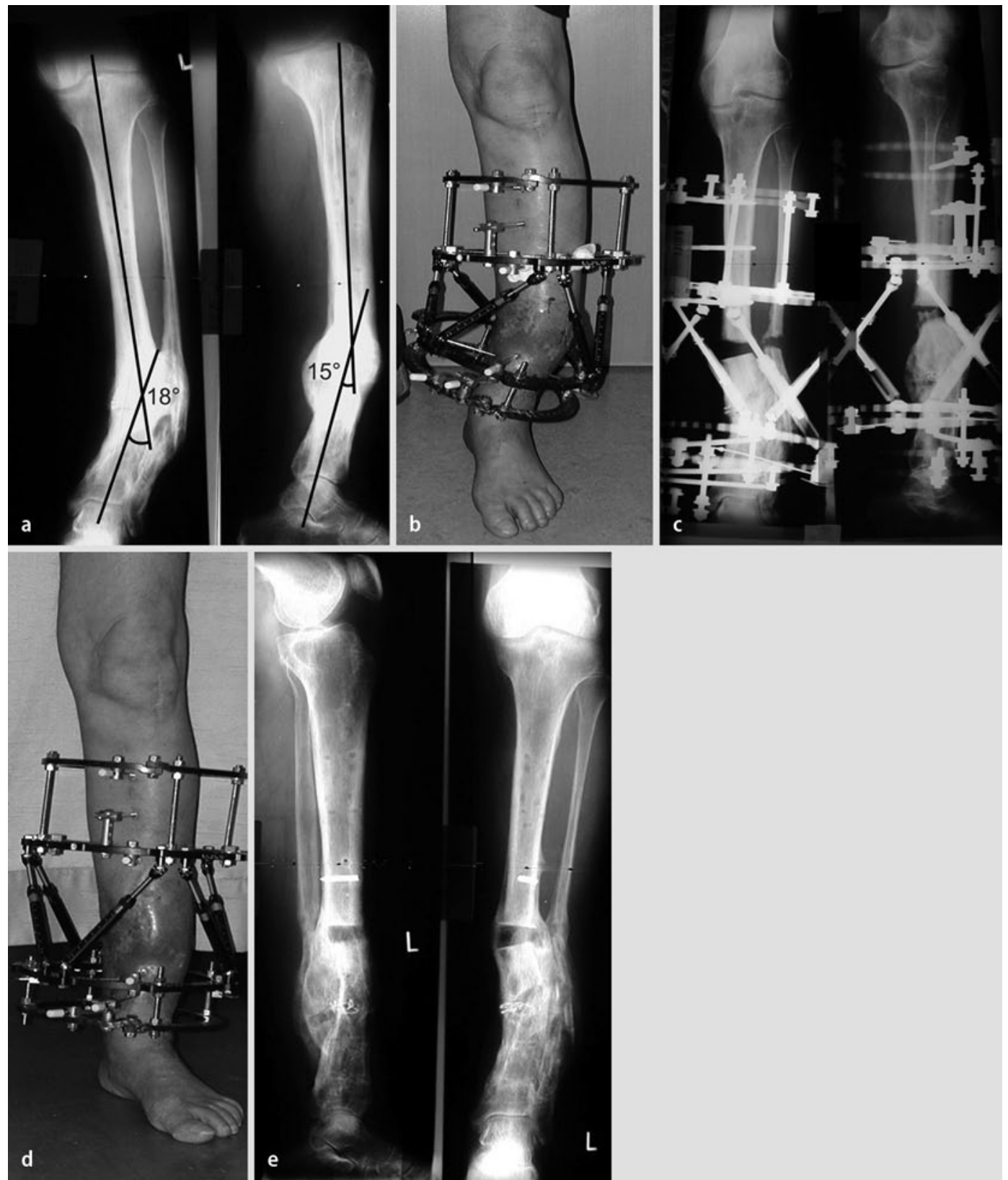

Abb. $1<57$ Jahre alter, männlicher Patient mit offener distaler Unterschenkelfraktur, IDDM (,,insulin demanding diabetes mellitus"), schwerem Nikotinabusus, a Zustand nach Versorgung mit UTN (unaufgebohrter Tibianagel), Weichteilinfekt, Osteitis, monolateralem Fixateur externe: kombinierte Fehlstellung der distalen Tibia: Varus $18^{\circ}$, Rekurvation $15^{\circ}$, b klinisches Bild des Unterschenkels mit angelegtem, die Fehlstellung quasi imitierendem Hexapoden (Taylor-Spatial-Frame); beachte kritische Weichteilverhältnisse, u. a. mit Stauungsdermatitis, c Röntgenbild am Ende der Korrektur zu Beginn der Konsolidierung, $\mathbf{d}$ klinisches Bild am Ende der Korrektur, e Röntgenbild nach Abbau des Fixateur externe mit gebrochenem, verbliebenem Fixateurpin

den. Maßgeblich ist die CT-Diagnostik für die exakte Bezifferung von Rotationsfehlstellungen. Bei Kindern gilt es zu überlegen, ob die Rotationsfehlstellung per Sonographie bestimmt wird, um Strahlung einzusparen. Beschrieben ist hier v. a. die Methode nach Keppler et al. [4], die sich, trotz der v. a. bei Kindern wichtigen Reduktion der Strahlenbelastung, aufgrund der allgemeinen Verfügbarkeit der CT bisher klinisch nicht durchsetzte.

\section{Therapie}

\section{Indikationsstellung}

Ob Winkelgrade bzw. Längendifferenzen korrekturpflichtig sind, ist aufgrund geringer Fallzahlen hinsichtlich des Followup und völlig unterschiedlicher Rahmenbedingungen bei verschiedenen Patienten nur mit geringer Evidenz belegt. Hinsichtlich der Vorhersage, ob eine Achsfehlstellung außer etwaigen ästhetischen Problemen zukünftige Beschwerden bereiten wird, hilft am Kniegelenk die Bestimmung der Miculicz-Linie. Es ist bekannt, dass es bei geringfügigen Änderungen der Trageachse zur signifikanten Erhöhung des Anpressdrucks und somit der Arthroseentwicklung im jeweils betroffenen Kniegelenkkompartiment kommt. Beinlängendifferenzen führen zur LWS-Skoliose (LWS: Lendenwirbelsäule), Genu recurvatum und antecurvatum zur Patellaluxation bzw. Hyperkompression. Torsionale Fehlstellungen können beim Laufen und z. B. beim Fahrradfahren stören, insbesondere wenn der 0-Durchgang nicht möglich ist. Weitere Kriterien können kosmetischer Art sein. Zusätzlich müssen die Knochenqualität, das Alter des Patien- 
ten und etwaige Risikofaktoren bei der Indikationsstellung einbezogen werden [3, 9]. Insbesondere bei posttraumatischen Achsfehlstellungen und Deformitäten gilt es immer, vorgeschädigtes Gewebe/Knochen mit zu berücksichtigen, sodass hier die Evidenz noch geringer ist, wie beispielsweise bei juvenilen kindlichen Skelettmissbildungen. Unter anderem auf den bereits oben zitierten Literaturstellen beruhend sind die in $\mathbf{0}$ Tab. 1 aufgeführten Maßzahlen hinweisgebend.

\section{Operationsplanung}

Es werden anatomische Landmarken festgelegt und zeichnerisch oder mittels eines computergestützten Planungsprogramms die Ist- und Soll-Analyse durchgeführt. Die technische Durchführung der Planung ist an anderer Stelle $[6,8,9]$ ausführlich beschrieben und soll nicht Gegenstand des vorliegenden Beitrags sein. Mit Hilfe des Korrekturziels werden die Osteotomiehöhe und der Korrekturwinkel festgelegt.

\section{Operative Technik}

Prinzipiell stehen die akute sowie die allmähliche Korrektur beispielsweise über Kallusdistraktion mittels Fixateur externe zur Verfügung. Bei der einzeitigen Korrektur werden meist herkömmliche Osteosyntheseimplantate verwendet (Marknagel, Platte). Bei den mehrzeitigen oder allmählichen Korrekturverfahren wird in der Regel auf einen Fixateur externe zurückgegriffen. Eine Ausnahme hiervon stellt die Extremitätenverlängerung mittels Verlängerungsnagel dar.

\section{Osteotomieformen}

Eine Vielzahl von Osteotomietechniken ist beschrieben. Quere Osteotomien kommen bei der Kallusdistraktion und bei Torsionsfehlern zum Einsatz. Die Keilosteotomie kann entweder additiv oder subtraktiv durchgeführt werden und ist bei Achsfehlern, Translationsfehlern und auch bei kombinierten Torsionsfehlern indiziert. Die Dom- und die Pendelosteotomie zeichnen sich dadurch aus, dass sie nahezu längenneutral sind, sie finden bei Achsfehlern Verwendung. Eine ähnliche Technik stellt die sog. Schweibenwischer-

Trauma Berufskrankh 2014 - 16[Suppl 2]:173-179 DOI 10.1007/s10039-013-2018-6

c) Springer-Verlag Berlin Heidelberg 2013

\section{H.J. Bail · K. van Scherpenzeel Rekonstruktion von posttraumatischen Beindeformitäten}

\section{Zusammenfassung}

Hintergrund. Bei posttraumatischen Fehlstellungen gibt es hinsichtlich der Korrekturgrenzen wenig Evidenz. Es existieren dennoch Grenzwerte, die v. a. beim jungen Patienten spätere Beschwerden erwarten lassen.

Planung und Therapiewahl. Eine gründliche präoperative klinische und radiologische Analyse ist unabdingbar, gefolgt von einer zeichnerischen Planung. Nicht selten sind die Weichteile posttraumatisch so verändert, dass die Stellungskorrektur mittels Fixateur externe zu bevorzugen ist. Bei multidimensionalen Umstellungen bewährte sich der Hexapode. In einem hohen Prozentsatz der
Fälle kann damit das Korrekturziel erreicht werden. Wenn nur eine Verlängerung durchgeführt werden muss, sind intramedulläre Verlängerungsnägel für den Patienten komfortabler, oft ist auch das kosmetische Ergebnis besser. Mittlerweile gibt es neue, besser steuerbare, allgemein verfügbare intramedulläre Verlängerungsnägel, die eine Reduktion der Komplikationsrate bei diesem Verfahren erwarten lassen.

\section{Schlüsselwörter}

Fehlstellung · Fixateur externe . Intramedullärer Verlängerungsnagel . Hexapode - Osteotomie

\section{Reconstruction of posttraumatic leg deformities}

\section{Abstract}

Background. Regarding the indications for correction of posttraumatic malalignment little evidence is available; however, threshold values exist where it can be expected that especially younger patients can have long-term problems.

Planning and therapy selection. Prior to surgery a thorough clinical and radiological analysis followed by a graphical planning has to be performed. In many cases the soft tissue has so much posttraumatic alteration that an external fixator must be used. Regarding multidirectional correction the hexapod fixator has been shown to reliably ensure the desired aim in a high proportion of cases. If only limb lengthening has to be performed, intramedullary lengthening nails are better for patient comfort and can have a better cosmetic result. Recently better controllable lengthening nails have become available which may lead to a reduction in the complication rate of this technique.

\section{Keywords}

Bone malalignment - External fixator . Intramedullary distraction nail $\cdot$ Hexapod fixator - Osteotomy osteotomie dar, bei der über eine schräge Osteotomie durch einen festen Drehpunkt ebenfalls eine fast längenneutrale Achskorrektur durchgeführt werden kann. Treppenförmige Osteotomien sind durch ihre große Kontaktfläche zusammen mit eingepassten kortikospongiösen Spänen gut für die Akutverlängerung, besonders am Femur, geeignet. Limitiert ist diese Technik durch die Dehnbarkeit der Weichteile, insbesondere der Nn. ischiadicus und femoralis.

\section{Multiplanare Korrektur mit dem Hexapodenfixateur}

Nach erfolgter korrekter Achsanalyse bietet der Hexapode eine für den Operateur komfortable Möglichkeit, v. a. multiplanare Fehlstellungen zu korrigieren. Mit einer Konstruktion von 6 schräg zueinander stehenden Teleskopstangen ähnelt er einem Flugsimulator, der ebenfalls alle möglichen Stellungen im Raum einnehmen kann.

Bei der Korrektur mit dem Hexapodenfixateur wird einem Computerprogramm mitgeteilt, wie die Knochenfragmente im Fixateur positioniert sind. Es wird ein Punkt in der Osteotomiefläche definiert, der als Ursprungspunkt (Origin) bezeichnet wird. Bezug nehmend auf diesen wird das 2. Fragment (meist das distale Fragment) durch den Fixateur positioniert. Der Hexapode erspart dabei aufwendige Ilizarov-Fixateur-Montagen, die oft nur für die Korrektur in einer oder maximal 2 Ebenen konstruierbar sind. In dem Computerprogramm wird ebenfalls 

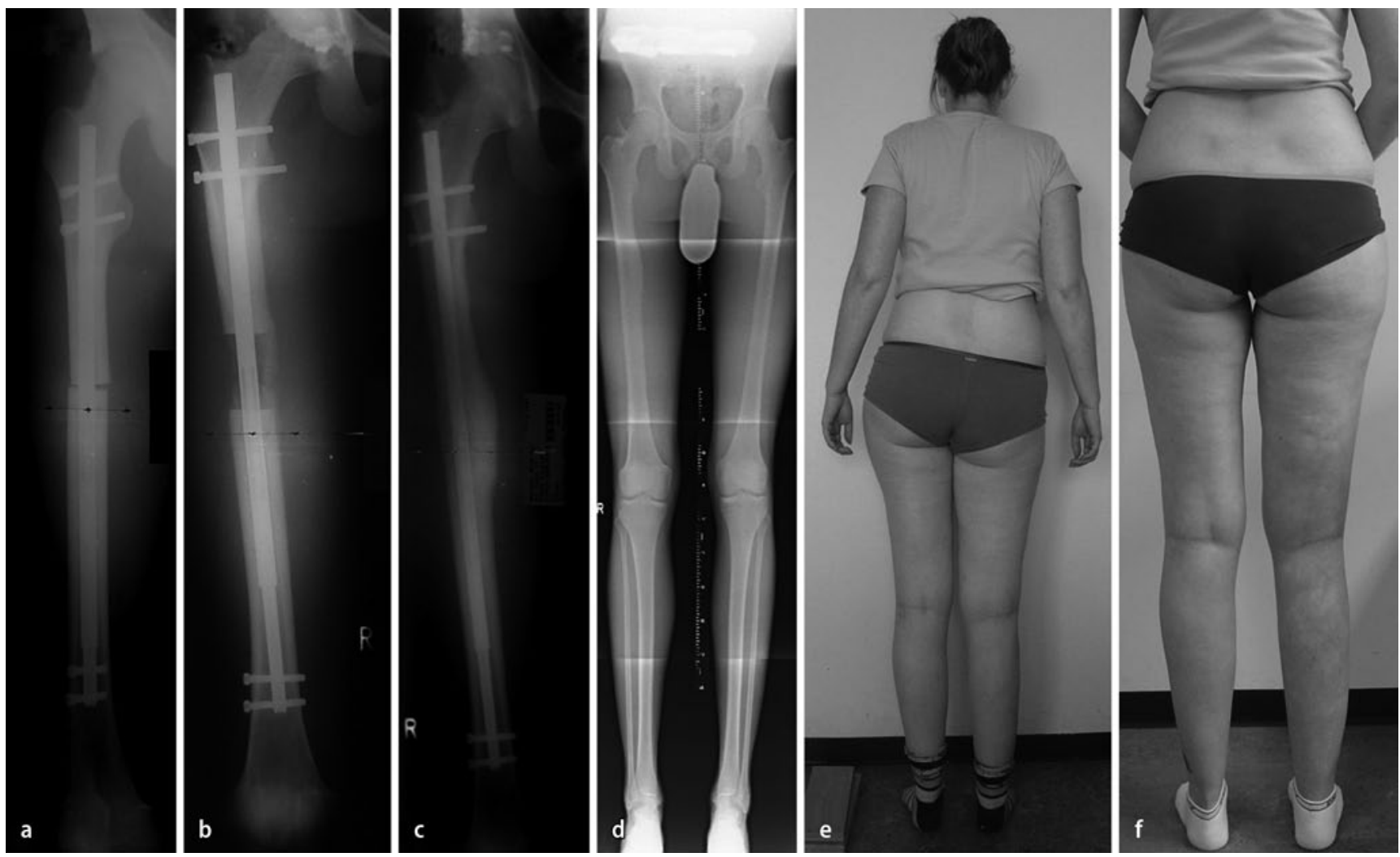

Abb. $2 \Delta$ 19-jährige Patientin mit posttraumatischer Beinlängendifferenz von $4 \mathrm{~cm}$ rechts, a-d röntgenologische Befunde, e,f klinisches Bild, a-c Oberschenkel a.-p., a nach 4 Tagen: ISKD-Nagel (ISKD:, intramedullary skeletal kinetic distractor") implantiert, Osteotomie proximale Diaphyse, b nach 2,5 Monaten: nach erfolgter Verlängerung, während der Konsolidierung: mediale Kallusbildung im Distraktionsspalt, c nach 7 Monaten: nach Konsolidierung vor der Implantatentfernung, $\mathbf{d}$ nach 18 Monaten: gleiche Beinlänge nach Implantatentfernung in der Becken-Bein-Ganzaufnahme im Stehen, e Beinlängendifferenz von $4 \mathrm{~cm}$ rechts, $\mathbf{f}$ Befund nach erfolgtem Beinlängenausgleich

eingegeben, wie groß die Distraktionsrate sein soll. Anschließend wird eine Liste mit Maßzahlen errechnet, anhand derer der Patient selbst die Länge der Teleskopstangen verändert und damit die Stellungskorrektur des jeweiligen Knochens durchführt. Dem System kann auch mitgeteilt werden, wo sich im Verhältnis zum Origin gefährdete Strukturen (z. B. N. peronaeus) befinden. Die eingegebene Distraktionsrate wird auf diese Struktur bezogen und an dieser nicht überschritten. Somit können Nerventraktionsschäden vermindert werden.

Die Korrektur mittels Hexapode und somit Fixateur externe ist insbesondere bei kritischen Weichteilverhältnissen, wie sie oft bei posttraumatischen Fehlstellungen vorliegen, geeignet ( $\mathbf{D}$ Abb. 1). Nach Abschluss der Korrektur werden die Achsen erneut analysiert. Durch den Widerstand des Gewebes kommt es immer zur Verbiegung der Ilizarov-Drähte oder Fixateurpins und zur Verwringung des Ge- samtkonstrukts. In solchen Fällen sind problemlos eine oder auch eine zweite Nachkorrektur möglich.

In unserem eigenen Krankengut wurden 42 Fälle anhand der Röntgenbilder nachuntersucht. Die Patienten waren 9 bis 64 Jahre alt, 32 waren männlich. Im Schnitt wurden bei der Erstkorrektur um etwa $1 \mathrm{~cm}$ verlängert, eine Translation von $3 \mathrm{~mm}$ durchgeführt, ad axim $26^{\circ}$ korrigiert und $8^{\circ}$ Rotation ausgeglichen. Durchschnittlich kam es zu folgenden Abweichungen vom ursprünglichen Korrekturziel: 10\% bei der Verlängerung bei der Erstkorrektur, 19\% bei der Translation, $18,5 \%$ bei der Korrektur ad axim und $21 \%$ bei der Rotationskorrektur. In allen Fällen konnte das geplante Korrekturziel mittels Zweitkorrektur, die im Schnitt 5,5 Tage dauerte (- Tab. 2), erreicht werden. Insgesamt betrug die durchschnittliche Korrekturdauer 44 Tage, die Konsolidierungszeit 150 Tage (• Tab. 3). Diese Zahlen zeigen, dass der Hexapode v. a. zur Achs- und zur Rotationskorrektur verwendet wurde, die Verlängerung und die Korrektur der Translation spielten eine nachgeordnete Rolle ( $\bullet$ Tab.3).

In einer Publikation von Manner et al. [5] wurden 208 Korrekturen an $155 \mathrm{~Pa}$ tienten durchgeführt. Mit dem IlizarovRingfixateur wurde 79-mal, mit dem Taylor-Special-Frame (Hexapode) 129-mal korrigiert. Das Ziel wurde in $91 \%$ der mit Taylor-Spatial-Frame gegenüber nur 56\% der mit Ilizarov-Ringfixateuren versorgten Fälle erreicht. Insbesondere ergaben sich exzellente Resultate für den TaylorSpatial-Frame, wenn in mehr als einer Dimension korrigiert wurde. Somit wurde das Korrekturziel bei 91\% der Patienten erreicht, wenn dreidimensional korrigiert wurde, während mit dem Ilizarov-Ringfixateur bei diesen komplexen Korrekturen nur in etwa 30\% das angestrebte Korrekturziel bewirkt werden konnte. Die Autoren schlossen aus ihren Ergebnissen, dass der Taylor-Spatial-Frame eine höhere Prä- 


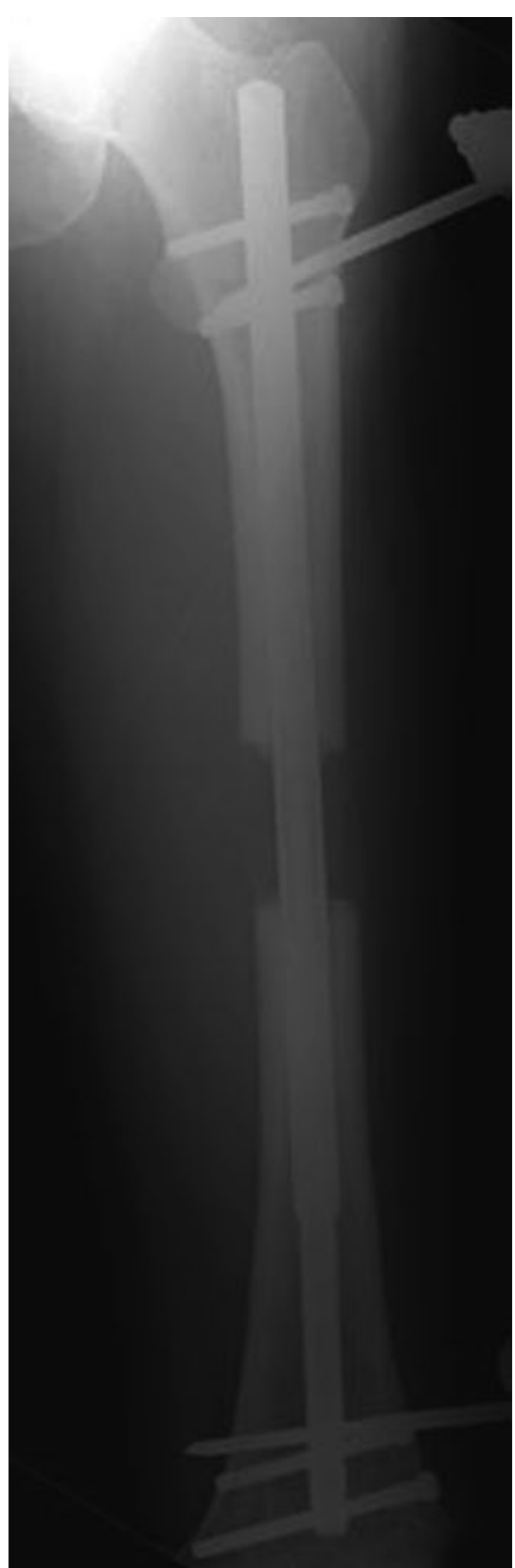

Abb. 3 А ISKD-Nagel (ISKD: ,intramedullary skeletal kinetic distractor") in situ mit angebrachtem Fixateur externe zum Abbremsen einer übermäßigen Verlängerung

zision bei der Korrektur von Fehlstellungen ermöglicht als der Ilizarov-Ringfixateur [5].

\section{Verlängerung mittels intramedullärem Verlängerungsnagel}

Bei den multidimensionalen Fehlstellungen bietet ein externer Fixateur große Vorteile. Bei eindimensionalen Korrekturen und hier insbesondere bei den Verlän-

Tab. 2 Aufsummierte Daten zur Korrektur mit dem Hexapoden ${ }^{\text {a }}$

\begin{tabular}{|lllllll}
\hline & $\begin{array}{l}\text { Kurz/ } \\
\text { lang }\end{array}$ & Translation & $\begin{array}{l}\text { Ad } \\
\text { axim }\end{array}$ & Rotation & $\begin{array}{l}\text { Korrektur- } \\
\text { dauer }\end{array}$ & $\begin{array}{l}\text { Konsolidierungs- } \\
\text { zeit }\end{array}$ \\
\hline Erstkorrektur & $306 \mathrm{~mm}$ & $93 \mathrm{~mm}$ & $933^{\circ}$ & $273^{\circ}$ & 1602 Tage & 6273 Tage \\
\hline Zweitkorrektur & $27 \mathrm{~mm}$ & $18 \mathrm{~mm}$ & $172^{\circ}$ & $58^{\circ}$ & 288 Tage & \\
\hline Abweichung & $9 \%$ & $19 \%$ & $18,5 \%$ & $21 \%$ & $\begin{array}{l}\text { Durchschnitt: } \\
\text { 5,5 Tage }\end{array}$ & \\
\hline $\begin{array}{l}\text { aBei 42 Patienten (9 bis 64 Jahre alt, 32 männlich), bProzentuale Abweichung vom Korrekturziel nach der Erst- } \\
\text { korrektur }\end{array}$ & & & & & & \\
\hline
\end{tabular}

Tab. 3 Durchschnittliche Korrekturausmaße mit dem Hexapoden ${ }^{2}$

\begin{tabular}{|lllllll}
\hline & $\begin{array}{l}\text { Kurz/ } \\
\text { lang } \\
(\mathrm{mm})\end{array}$ & $\begin{array}{l}\text { Translation } \\
(\mathrm{mm})\end{array}$ & $\begin{array}{l}\text { Ad } \\
\text { axim } \\
\left({ }^{\circ}\right)\end{array}$ & $\begin{array}{l}\text { Rotation } \\
\left({ }^{\circ}\right)\end{array}$ & $\begin{array}{l}\text { Korrek- } \\
\text { turdauer } \\
(\text { Tage })\end{array}$ & $\begin{array}{l}\text { Konsoli- } \\
\text { dierungs- } \\
\text { zeit (Tage) }\end{array}$ \\
\hline $\begin{array}{l}\text { Ausmaß der Korrektur } \\
\text { (Erst- plus Zweitkorrektur) }\end{array}$ & 8 & 3 & 26 & 8 & 44 & 150 \\
\hline aBei 42 Patienten (9 bis 64 Jahre alt, 32 männlich) & & & & & \\
\hline
\end{tabular}

gerungen jedoch sind die hohe Versatilität und Multidirektionalität beispielsweise des Hexapodenfixateurs nicht notwendig. Eine reine Verlängerung kann theoretisch über einen intramedullären Kraftträger vorgenommen werden.

Ein intramedullärer Verlängerungsnagel, der weite klinische Verbreitung erfuhr, ist der ISKD-Nagel (ISKD: ,intramedullary skeletal kinetic distractor“; Fa. Orthofix, Ottobrunn). Er benötigt zum Transport 3-9 ${ }^{\circ}$ Rotation der Extremität, welche bei der normalen Mobilisierung des $\mathrm{Pa}$ tienten erreicht wird und nicht gesondert manuell durch Drehen an der Extremität bewerkstelligt werden muss. Die jeweilige Distraktionsgeschwindigkeit wird durch einen Magneten an ein transkutan positioniertes Messgerät mitgeteilt. Mit diesem Nagel konnten kosmetisch ansprechende Verlängerungsprozeduren v. a. am Femur vorgenommen werden (• Abb. 2). Problematisch war jedoch, dass der Nagel mit Beginn der Distraktion des Öfteren zu schnell transportierte und auch der Verlängerungsprozess schlecht beeinflusst werden konnte. So musste im eigenen $\mathrm{Pa}$ tientengut 2-mal ein Fixateur externe angebracht werden, um ein Abreißen des Regenerats und ein übermäßiges Dehnen der Weichteile einschließlich der Nerven zu verhindern ( $\bullet$ Abb. 3). In einer Publikation von Simpson et al. [7] von 2009 wurde bei 33 Patienten in 24\% der Fälle eine zu schnelle Distraktion festgestellt. Bei 21\% sistierte der Transport. Dies bedeutet eine schwerwiegende implantatbezogene Komplikation bei nahezu 50\% der Patienten. Zusätzlich kam es in dieser Patientengruppe bei 2 Patienten zum Implantatversagen [7]. Im Vergleich dazu gehen der Albizzia-Nagel mit einer Implantatversagensrate von etwa 10\% [2] und der Fitbone-Nagel von etwa $17 \%$ einher [1]. Diese beiden Verlängerungsnägel erfordern entweder eine umfangreichere, manuell durchzuführende Rotation, die nicht so leicht von selbst die Verlängerung auslöst (Albizzia-Nagel), oder aber eine externe elektromagnetische Verlängerungsvorrichtung, die nur beim Anlegen derselben transportiert (Fitbone-Nagel). Auf Basis der berichteten Komplikationsraten wurde der ISKD-Nagel Ende 2012 vorübergehend vom Markt genommen.

Seit kurzem steht ein Verlängerungsnagel zur Verfügung, der vergleichbar mit dem Fitbone-Nagel die Verlängerung über eine externe magnetische Steuerung bewirkt (- Abb. 4, 5). Dieser sog. Precice-Nagel (Fa. Orthovative, Gmund) wird z. B. von Paley (http://www.paleyinstitute.org) regelhaft für kosmetische Verlängerungen eingesetzt. Nach seinen Angaben sind die Komplikationsraten dabei relativ gering. Die kosmetische Verlängerung zur Größenzunahme spielt in Deutschland bislang eine vergleichsweise geringe Rolle. International, insbesondere in den USA, nimmt die Anzahl kosmetischer Verlängerungen jedoch rapide $\mathrm{zu}$ [zum Preis von 170.000 US\$ bei bilateraler Femur- und Tibiaverlängerung (http:// www.paleyinstitute.org)]. 


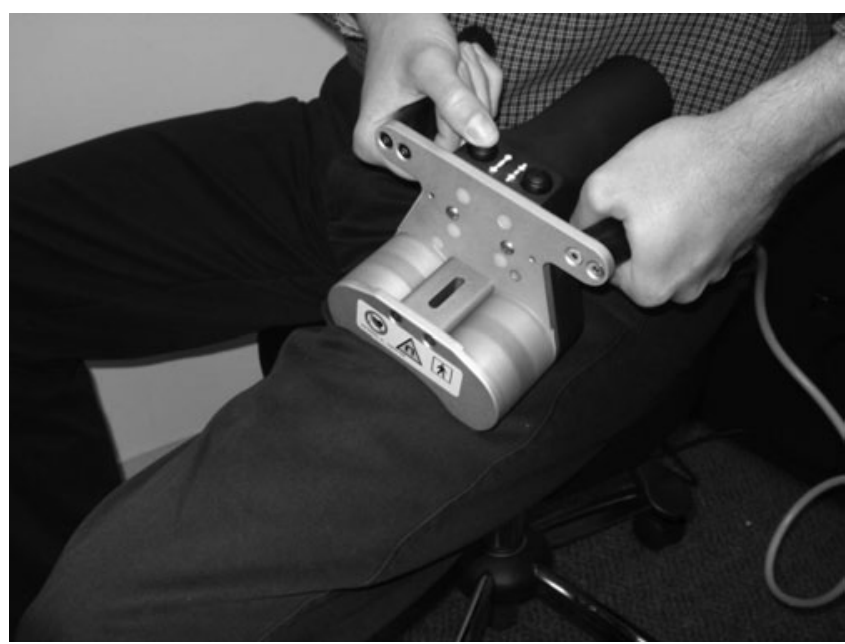

Abb. $4<$ Verlängerungsvorgang beim Precice-Nagel: Patient hält externen Antrieb an den Oberschenkel; rotierende Magneten rotieren den im $\mathrm{Na}$ gel befindlichen Magneten (mit freundl. Genehmigung der Fa. Orthovative, Gmund)

\section{Resümee}

Bei der Beurteilung, ob posttraumatische Fehlstellungen korrigiert werden sollen, sind die Beschwerden des Patienten ausschlaggebend. Zusätzlich gibt es, bei zwar geringer Evidenz, Grenzwerte, bei denen aufgrund zu erwartender nachfolgender Beschwerden, insbesondere bei jungen Patienten, eine Fehlstellungskorrektur durchgeführt werden soll (z. B. $>2 \mathrm{~cm}$ Beinlängendifferenz beim jungen Patienten oder fehlender rotatorischer 0-Durchgang).

Die operativen Prinzipien der Korrektur posttraumatischer Deformitäten ähneln denen bei Deformitäten anderer Genese.

Eine gründliche präoperative Analyse, sowohl klinisch als auch mit Bildgebung, ist notwendig. Planungsprogramme können dabei hilfreich sein. Aufgrund posttraumatisch kompromittierter Weichteile ist häufig der Einsatz eines externen Fixateurs indiziert. Hinsichtlich mehrdimensionaler Umstellungen bewährte sich klinisch der Hexapode. Bei diesem Verfahren kann auch bei multidimensionalen Umstellungen in einem hohen Prozentsatz der Fälle das Korrekturziel erreicht werden.

Bei der singulären Extremitätenverlängerung können intramedulläre Verlängerungsnägel eingesetzt werden. Hierbei bieten die von extern steuerbaren Nägel möglicherweise deutliche Vorteile gegenüber den durch mechanische Belastung transportierenden Konstrukten. Dies lässt erwarten, dass das kosmetisch anspre- chende und für den Patienten komfortable Verfahren der intramedullären Verlängerung in der Zukunft komplikationsärmer wird.

\section{Fazit für die Praxis}

- Die Indikation zur Korrektur posttraumatischer Fehlstellungen wird abhängig von den Beschwerden des Patienten und anhand von Grenzwerten gestellt.

- Die operativen Prinzipien der Deformitätenkorrektur ähneln denen bei Deformitäten anderer Genese.

- Präoperativ ist eine gründliche klinische und bildgebende Diagnostik unverzichtbar.

- Aufgrund posttraumatisch kompromittierter Weichteile ist häufig die Verwendung eines externen Fixateurs indiziert.

- Bei mehrdimensionalen Umstellungen bewährte sich der Hexapode, mit welchem in einem hohen Prozentsatz der Fälle das Korrekturziel erreicht werden kann.

- Die singuläre Extremitätenverlängerung ist mittels intramedullärer Verlängerungsnägel möglich.

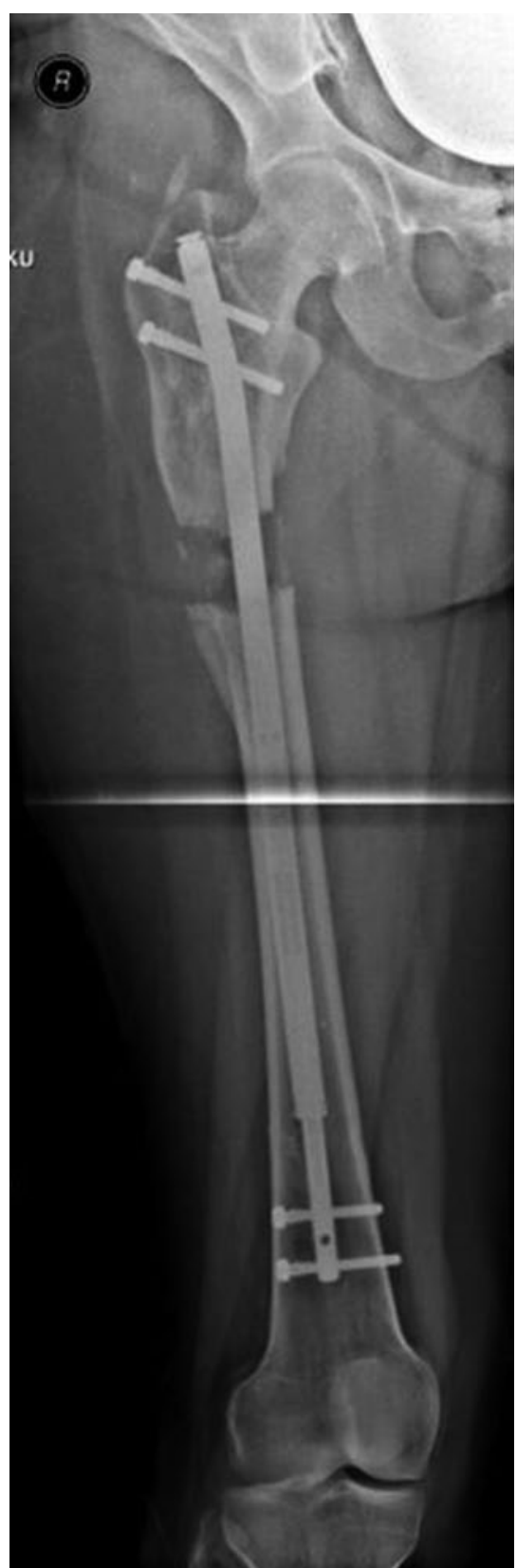

Abb. $5 \Delta$ Femur a.-p. mit einliegendem Precice-Nagel; 28-jährige Patientin mit posttraumatischer Beinlängendifferenz von $3 \mathrm{~cm}$ 


\section{Korrespondenzadresse}

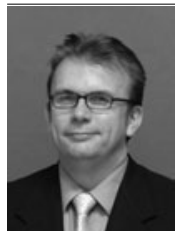

PD Dr. H.J. Bail

Klinik für Unfall- und

Orthopädische Chirurgie,

Klinikum Nürnberg,

Breslauer Straße 201,

90471 Nürnberg

hermann-josef.bail@

klinikum-nuernberg.de

\section{Einhaltung ethischer Richtlinien}

Interessenkonflikt. H.J.Bail und K. van Scherpenzeel geben an, dass kein Interessenkonflikt besteht.

Dieser Beitrag beinhaltet keine Studien an Menschen oder Tieren.

The supplement containing this article is not sponsored by industry.

\section{Literatur}

1. Baumgart R, Betz A, Schweiberer L (1997) A fully implantable motorized intramedullary nail for limb lengthening and bone transport. Clin Orthop 343:135-143

2. Guichet JM, Deromedis B, Donnan LT et al (2003) Gradual femoral lengthening with the Albizzia intramedullary nail. J Bone Joint Surg Am 85-A:838848

3. Keppler P, Kinzl L (2006) Diagnose der posttraumatischen Beindeformitäten. Trauma Berufskrankh 8:303-315

4. Keppler P, Strecker W, Kinzl L et al (1999) Sonographic imaging of leg geometry. Orthopade 28(12):1015-1022

5. Manner HM, Huebl M, Radler C et al (2007) Accuracy of complex lower-limb deformity correction with external fixation: a comparison of the Taylor Spatial Frame with the llizarov Ringfixator. J Child Orthop 1:55-61

6. Paley T (2002) Principles of deformity correction. Springer, Berlin Heidelberg New York

7. Simpson AH, Shalaby H, Keenan G (2009) Femoral lengthening with the intramedullary skeletal kinetic distractor. J Bone Joint Surg Br 91(7):955-961

8. Strecker W (2006) Planerische Analyse kniegelenknaher Beinachsabweichungen. Oper Orthop Traumatol 18:259-272

9. Strecker W, Keppler P (2002) Analyse und Korrektur von Beindeformitäten. Unfallchirurg 73:811829 